\title{
The Doppler Effect and the Anisotropy of the Speed of Light
}

\author{
Michał Drągowski ${ }^{1}$ (D) - Marta Włodarczyk ${ }^{1}$
}

Received: 3 October 2019 / Accepted: 25 February 2020 / Published online: 4 April 2020

(c) The Author(s) 2020

\begin{abstract}
Fundamental incompatibility arises at the interface of quantum mechanics and the special theory of relativity with Einstein synchronization, in which simultaneity is not absolute. It has, however, been shown that a relativistic theory preserving absolute simultaneity allows to formulate Lorentz-covariant quantum theory, at a price of introducing a preferred frame of reference manifesting itself in a directional anisotropy of the speed of light. We show that a supposed method of distinguishing between these two theories based on the Doppler effect is insensitive to this anisotropy. Both theories are indistinguishable if only kinematic effects for light or subluminal signals are considered.
\end{abstract}

Keywords Special relativity $\cdot$ Preferred frame $\cdot$ Speed of light $\cdot$ Mössbauer rotor

\section{Introduction}

The assumption of isotropy of the speed of light was postulated by Einstein at the foundation of the special theory of relativity (STR). It has not yet been verified experimentally, since only the two-way speed of light can be measured directly (e.g., with the time-of-flight method). Theories violating the constancy of the speed of light measured over a closed path would yield different results than STR for a large class of closed path experiments, such as the Michelson-Morley experiment [1], which over time have been performed to a very high precision. Meanwhile, theories introducing a directional anisotropy but conserving the value of the speed of light measured over a closed path, would yield the same results as STR for any direct or closed path measurement.

On the other hand, the one-way velocity of light cannot be measured directly (the clock synchronization procedure necessary for such measurement is essentially equivalent to closing the path). One could potentially detect a directional anisotropy

Michał Drągowski

mdragowski@fuw.edu.pl

1 Faculty of Physics, University of Warsaw, Pasteura 5, 02-093 Warsaw, Poland 
only in an indirect measurement, involving processes sensitive to the one-way velocity of light.

Therefore, here we investigate the Doppler effect for electromagnetic waves, which intuitively could be sensitive to the one-way velocity of light, since it involves a light signal sent only one-way from an emitter to a receiver. We demonstrate that a theory preserving both absolute simultaneity and the constancy of the speed of light measured over a closed path is indistinguishable from STR by means of this effect.

We also make reference to available experimental evidence regarding the directional anisotropy of the Doppler effect. Our result proves that the upper limits on the velocity of Earth with respect to a supposed preferred frame of reference, derived from experiments investigating directional dependence of the effect, are not conclusive, since a null effect is predicted both by STR and a preferred frame theory preserving absolute simultaneity.

In view of our result and the nonexistence of different experimental tests addressing this problem, the question of directional anisotropy of the speed of light still remains valid. The experimental verification of this fundamental assumption should be carried out by means of different (non-kinematic) effects sensitive to the one-way velocity of light. It is particularly important in view of the recent revival of interest in theories introducing a preferred frame of reference, related to the aims at quantization of gravity $[2,3]$. Additionally, the exceptionally desirable properties of the preferred frame theories for the description of quantum phenomena are mentioned shortly in the subsequent section.

\section{Absolute Simultaneity}

It has been noted by several authors (see, e.g., Reichenbach [4]) that the synchronization procedure in special relativity is only a convention. The one-way velocity of light is synchronization dependent, but this effect is not physically observable within STR, making all synchronizations equivalent as long as kinematic effects for light or subluminal signals are considered. A choice of a different synchronization convention would change the mathematical formalism (transformation equations) but not the results of physical measurements. It is only the existence of particles faster than light that would allow for absolute synchronization of distant clocks and a direct measurement of the one-way velocity of light that would distinguish a particular synchronization convention.

Thanks to its symmetry, related to the relativity principle, the Einstein's formulation results in particularly elegant transformation laws, but is irreconcilable with any theory that is non-local (quantum mechanics) nor does it allow for a consistent description of super-luminal particles (tachyons). Not only the relative simultaneity of events in STR has been perceived as counterintuitive, but most importantly mixing of the space and time coordinates makes STR difficult to interface with the quantum theory, in which time is an independent parameter. Historically, this led to the formulation of the well known Einstein-Podolsky-Rosen paradox [5], related to the apparent causality violation during the reduction of the wave function of an entangled state, caused by the relative simultaneity of events in STR. Among all 
theories special attention has been paid to those preserving absolute simultaneity, which overcome these limitations at a price of introducing a preferred frame of reference $(\mathrm{PF})$, leading to the directional dependence of the speed of light.

A very detailed description of a test theory preserving absolute simultaneity, together with references to experimental results, was presented in a series of papers by Mansouri and Sexl [6-8]. Modified transformation equations were obtained from the standard Lorentz transformation by eliminating the dependence on spatial position from the transformation of time component and modifying the time dilation and length contraction factors. As a result, this theory does not conserve the value of two-way speed of light ${ }^{1}$. Such theory is, however, disfavored in light of large experimental evidence from closed path experiments, as was advocated above. Nevertheless, the formalism introduced in that work has been used in many historical and present experiments (e.g., [9]).

Meanwhile, a relativistic theory fulfilling the requirements of preserving both absolute simultaneity and the constancy of the speed of light measured over a closed path has been described in the works of Rembieliński (e.g., [10-13]). The one-way velocity of light $\vec{c}(\vec{n})$ in a direction determined by a unit vector $\vec{n}$ must have the following form in order to preserve the constancy of the speed of light measured over a closed path ${ }^{2}$ :

$$
\vec{c}(\vec{n})=\frac{\vec{n}}{1+\vec{\varepsilon} \vec{n}},
$$

where the synchronization coefficient $\vec{\varepsilon}$ can take any value satisfying $\vec{\varepsilon}^{2}<1$. The Einstein synchronization procedure corresponds to $\vec{\varepsilon}=\overrightarrow{0}$, leading to $\vec{c}(\vec{n})=\vec{n}$. The relation between time in arbitrary synchronization $t$ and time in Einstein synchronization $t_{E}$ is $^{3}$ :

$$
t=t_{E}-\vec{\varepsilon} \vec{x}_{E}
$$

Taking into account (2) and the fact that the choice of synchronization does not affect spatial coordinates the generalized Lorentz transformation laws, from an inertial frame of reference $O$ to an inertial frame $O^{\prime}$, have the following form for arbitrary synchronization procedure:

$$
\begin{gathered}
t^{\prime}=\gamma(\vec{\varepsilon})\left[t\left(1+\left(\vec{\varepsilon}+\vec{\varepsilon}^{\prime}\right) \vec{V}\right)+\vec{x}\left(\vec{\varepsilon}-\vec{\varepsilon}^{\prime}-\left(1-\vec{\varepsilon}^{2}\right) \vec{V}\right)\right] \\
\vec{x}^{\prime}=\gamma(\vec{\varepsilon})(\vec{x}-\vec{V} t)
\end{gathered}
$$

\footnotetext{
1 Additionally, contrary to the statements of the authors, transformation matrix obtained by eliminating the dependence on spatial position from the transformation of time component, while leaving the rest of the transformation matrix unchanged, is not equivalent to the standard Lorentz transformation.

2 Throughout the paper we assume $c=1$ where $c$ denotes the average speed of light measured over a closed path.

${ }^{3}$ In all equations subscript ${ }_{E}$ denotes Einstein synchronization.
} 
with

$$
\gamma(\vec{\varepsilon})=\frac{1}{\sqrt{(1+\vec{\varepsilon} \vec{V})^{2}-(\vec{V})^{2}}},
$$

where $\vec{V}$ denotes the velocity of $O^{\prime}$ with respect to $O$, in the new synchronization. Note that $\vec{V}$ is always lower than the velocity of light in the corresponding direction, but can be greater than the average $c$. The synchronization coefficient in the frame $O^{\prime}$ is denoted by $\vec{\varepsilon}^{\prime}$.

Absolute simultaneity requirement implies that the spatial position does not participate in the transformation of the time component:

$$
\vec{\varepsilon}^{\prime}=\vec{\varepsilon}-\left(1-\vec{\varepsilon}^{2}\right) \vec{V},
$$

which leads to the transformation equations fulfilling the requirement of absolute simultaneity:

$$
\begin{gathered}
t^{\prime}=\gamma(\vec{\varepsilon})^{-1} t, \\
\vec{x}^{\prime}=\gamma(\vec{\varepsilon})(\vec{x}-\vec{V} t) .
\end{gathered}
$$

One can observe that a particular frame of reference, corresponding to $\vec{\varepsilon}=\overrightarrow{0}$, is distinguished within this formalism. This preferred frame of reference (PF) is the only reference frame in which the velocity of light in any direction takes the same value (equal to the average $c$ ). Thus, all equations should reduce to the standard STR form in PF.

Equation (4) is also the transformation equation for $\vec{\varepsilon}$. By assuming that the frame $O^{\prime}$ is the preferred frame of reference it can be solved to determine $\vec{\varepsilon}$ [11]:

$$
\vec{\varepsilon}=\frac{1}{2} \frac{\vec{v}}{\vec{v}^{2}}\left(\sqrt{1+4 \vec{v}^{2}}-1\right),
$$

where $\vec{v}$ is the velocity of PF measured in $O$. Taking into account that $\vec{v}$ is related to the corresponding four-velocity $u$ by $\vec{v}=\vec{u} / u^{0}$, and that the four-velocities are normalized to unity, $\vec{\varepsilon}$ can be rewritten as:

$$
\vec{\varepsilon}=u^{0} \vec{u} .
$$

It can be shown [12] that the factor $\vec{u}$ is always present in $\vec{\varepsilon}$; it is the parameter used by an observer in PF to distinguish between different inertial frames of reference. Meanwhile, $u^{0}$ is specific to absolute synchronization. Note that it is thus the only synchronization convention resulting in a covariant formalism.

The preferred frame theory of Rembieliński not only allows to describe superluminal particles [12], which would lead to causality violation in STR, but also to formulate Lorentz-covariant quantum theory [13], with covariant position and spin operators that do not exist in the relativistic quantum theory based on STR. In the 
following section we derive the formula for energy transformation between inertial frames of reference within this framework.

\section{Doppler Effect in Preferred Frame Mechanics}

As has been shown in the previous section, the position vectors are equal in absolute and Einstein synchronization, while time in both synchronizations is related by $t=t_{E}-u^{0} \vec{u} \vec{x}_{E}$, from which follows the equation for velocity conversion:

$$
\vec{V}=\frac{\vec{V}_{E}}{1-u^{0} \vec{u} \vec{V}_{E}}
$$

In general, contravariant four-vectors in both synchronizations are related by the following conversion matrix ${ }^{4}$ :

$$
T_{\nu}^{\mu}(u)=\left(\begin{array}{cc}
1 & -u^{0} \vec{u}^{T} \\
0 & I
\end{array}\right) .
$$

The covariant momentum four-vector in absolute synchronization, used in the transformation below, is as well related to the corresponding four-vector in Einstein synchronization by an appropriate conversion matrix $T_{\mu}{ }^{v}(u)=\left(T^{-1}\right)_{\mu}^{v}(u)[12]$ :

$$
T_{\mu}{ }^{v}(u)=\left(\begin{array}{cc}
1 & 0 \\
u^{0} \vec{u} & I
\end{array}\right) .
$$

The energy component is thus equal in both synchronizations, while the momentum covectors are related by $p=\underline{p}_{E}+u^{0} \vec{u}^{T} E$.

The transformation matrix for covariant momentum four-vectors, from an inertial frame of reference $O$ to another inertial frame $O^{\prime}$ moving with respect to $O$ with a four-velocity $W$, is [12]:

$$
D_{\mu}{ }^{\nu}(W, u)=\left(\begin{array}{cc}
W^{0} & \vec{W}^{T} \\
0 & I-\frac{\vec{W} \otimes \vec{W}^{T}}{W^{0}\left(1+\sqrt{1+\vec{W}^{2}}\right)}+\frac{u^{0}}{W^{0}} \vec{u} \otimes \vec{W}^{T}
\end{array}\right),
$$

and the relation between $W$ and the velocity $\vec{V}$ of $O^{\prime}$ measured in $O$ has the following form:

$$
\vec{V}=\frac{\vec{W}}{W^{0}}
$$

\footnotetext{
${ }^{4}$ In all equations superscript ${ }^{T}$ represents transposition.
} 


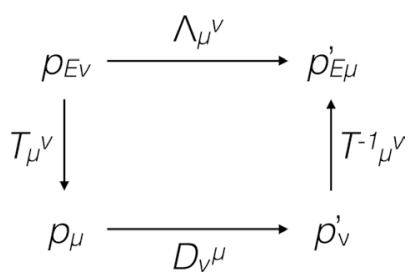

Fig. 1 Schematic representation of the transformations performed to calculate the energy change related to the Doppler effect, both in Einstein and absolute synchronization. $\Lambda$ denotes the standard Lorentz transformation, $D$-corresponding transformation in absolute synchronization, $T$-momenta conversions between the two synchronization conventions. Subscript $E$ denotes quantities in Einstein synchronization, in which physical measurements are performed

while $u$ is the four-velocity of the preferred frame of reference measured in $O$. The transformation matrix can be obtained from the standard Lorentz transformation using the conversion matrices shown above.

The Doppler effect refers to the change of energy (frequency) of electromagnetic radiation measured in a reference frame in motion with respect to the frame in which the radiation was emitted. The relation between energy measured in two reference frames $O$ and $O^{\prime}$ follows from (11):

$$
E^{\prime}=W^{0} E+\underset{\rightarrow}{\vec{W}}=W^{0}(E+\underset{\sim}{\vec{V}}) .
$$

$W^{0}$ can be calculated taking into account the fact that contravariant velocity fourvectors are normalized to unity:

$$
\left(W^{0}+u^{0} \vec{u} \vec{W}\right)^{2}-\vec{W}^{2}=1,
$$

using (12) to eliminate $\vec{W}$, which leads to the following equation:

$$
W^{0^{2}}=\frac{1}{\left(1+u^{0} \vec{u} \vec{V}\right)^{2}-\vec{V}^{2}} .
$$

The dependence of the metric tensor $g$ on $u$ has been accounted for above [12]:

$$
g^{\mu \nu}(u)=\left(\begin{array}{cc}
u^{0^{2}} & u^{0} \vec{u}^{T} \\
u^{0} \vec{u} & -I
\end{array}\right),
$$

which again can be derived from the Minkowski tensor using the conversion matrices $T$.

Using the momentum and velocity vectors in Einstein synchronization, (13) can be rewritten as:

$$
E^{\prime}=\frac{W^{0}}{1-u^{0} \vec{u} \vec{V}_{E}}\left(E+p_{-} \vec{V}_{E}\right)
$$

which, using (15), simplifies to the well known STR formula: 


$$
E^{\prime}=\frac{1}{\sqrt{1-\vec{V}_{E}^{2}}}\left(E+\underline{p}_{E} \vec{V}_{E}\right) .
$$

The above result is in line with the observation that the synchronization procedure is just a convention, and as such cannot affect the results of physical measurements. This concept is illustrated in Figure 1 showing that the calculations must lead to the same values of physically measurable quantities, regardless of the synchronization convention.

\section{Measurement of the Transverse Doppler Effect}

The transverse Doppler effect has been extensively investigated in a class of socalled Mössbauer rotor experiments. Resonance absorption of radiation by atomic nuclei was measured in a setup consisting of a source and absorber placed on a rotating disk. In most of the experiments the change in orientation of the experimental setup was not accounted for, thus only the average Doppler shift was measured and obviously no directional anisotropy could be detected. There were also a few such experiments [14-16], that investigated directional dependence of the effect.

It has been shown in the previous section that the null result of experiments investigating the Doppler effect does not exclude the anisotropy of the speed of light, since the formulas describing the energy change calculated with the absolute and Einstein synchronization are identical, even though the one-way velocities of light are not. As expected, no anisotropy was observed within the accuracy of the measurement in direction sensitive Mössbauer rotor experiments.

The authors of the experiments referenced above assumed the PF correction in the form $\vec{v}\left(\vec{V}_{1}-\vec{V}_{2}\right)$, which has been derived by Møller from non-relativistic ether theory [17], imposing an upper limit on $\vec{v}$ of the order of $10 \mathrm{~m} / \mathrm{s} . \vec{V}_{1}$ and $\vec{V}_{2}$ denote the velocity of the emitter and the absorber, respectively. It has been shown above that no such effect is predicted by a proper relativistic theory with a preferred frame. Both results are thus contradictory, since both the preferred frame and Lorentz transformations come down to the Galilean transformation in the non-relativistic limit. It will be shown below that the result of Møller coincides with the non-relativistic approximation of the exact relativistic formula only if the difference of momenta and velocities in different synchronization conventions is not accounted for ${ }^{5}$.

If the conversion between absolute and Einstein synchronization is omitted, Eq. (13), taking into account (15), takes the following form:

$$
E^{\prime}=\frac{1}{\sqrt{\left(1+u^{0} \vec{u} \vec{V}\right)^{2}-\vec{V}^{2}}}(E+\underset{\rightarrow}{\vec{V}}) .
$$

\footnotetext{
5 Precisely, the covariant momentum, which takes part in energy transformation, is different, while the kinematical momentum (contravariant vector), which is the quantity measured experimentally, is the same regardless of synchronization convention.
} 
In the preferred frame $\left(\vec{u} \rightarrow 0, u^{0} \rightarrow 1\right)$ this result reduces to the STR form (18), since $\vec{V} \rightarrow \vec{V}_{E}$ and $\underset{p}{\rightarrow} \rightarrow \underline{p}_{E}$ in PF.

Transverse effect refers to the Doppler shift observed with an absorber (emitter) moving with velocity $\vec{V}$ with respect to the source (absorber) of radiation, perpendicular to the radiation propagation direction. If one assumes, that in this case $p \vec{V}=0$ (instead of $\vec{V}_{E}$ being perpendicular to $\vec{p}_{E}$ ), (13) reduces to a very simple form:

$$
\frac{E^{\prime}}{E}=W^{0}=\frac{1}{\sqrt{\left(1+u^{0} \vec{u} \vec{V}\right)^{2}-\vec{V}^{2}}} .
$$

The resulting formula depends on two quantities only, $\vec{u}$ and $\vec{V}$, and $u^{0}$ can be expressed by $\vec{u}$ again taking into account that the four-velocity $u$ is normalized to unity:

$$
\frac{1}{u^{0^{2}}}-\vec{u}^{2}=1
$$

yealding:

$$
u^{0}=\sqrt{\frac{1}{1+\vec{u}^{2}}} .
$$

Obviously, in the PF $\left(\vec{u} \rightarrow 0, u^{0} \rightarrow 1\right)$ Eq. (20) as well reduces to the STR time dilation factor:

$$
\frac{E^{\prime}}{E}=\sqrt{\frac{1}{1-\vec{V}^{2}}}
$$

In many experiments both the emitter and absorber were in circular motion with respect to the laboratory frame. Thus, in a general case, two transformations (20) have to be combined yielding:

$$
\frac{E_{2}}{E_{1}}=\sqrt{\frac{\left(1+u^{0} \vec{u} \vec{V}_{1}\right)^{2}-\vec{V}_{1}^{2}}{\left(1+u^{0} \vec{u} \vec{V}_{2}\right)^{2}-\vec{V}_{2}^{2}}}
$$

where $\vec{V}_{1}$ and $\vec{V}_{2}$ denote the velocity of the emitter and absorber, respectively.

The Taylor series expansion of (24) in the semi-classical limit $\left(\vec{V}_{1} \ll 1, \vec{V}_{2} \ll 1\right.$ and $\vec{u} \ll 1)$ gives:

$$
\frac{E_{2}}{E_{1}} \approx \sqrt{\frac{1+2 \vec{u} \vec{V}_{1}-\vec{V}_{1}^{2}}{1+2 \vec{u} \vec{V}_{2}-\vec{V}_{2}^{2}}} \approx 1+\vec{u}\left(\vec{V}_{1}-\vec{V}_{2}\right)-\frac{1}{2}\left(\vec{V}_{1}^{2}-\vec{V}_{2}^{2}\right) .
$$

In the case when $\vec{V}_{1}=-\vec{V}_{2}$ the last term cancels out, thus to a first approximation the relative frequency change should be directly proportional to $\vec{u}$, while no effect 


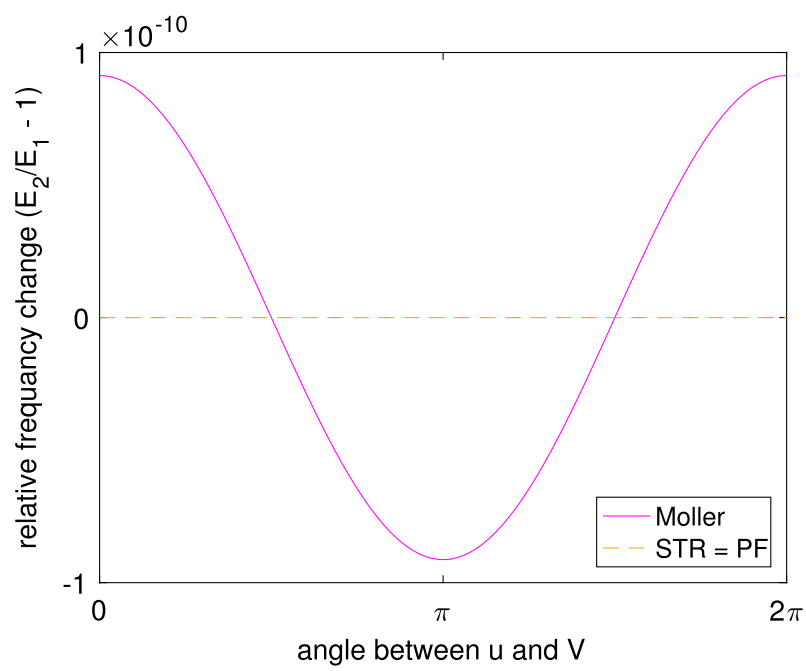

Fig. 2 The relative frequency change caused by the transverse Doppler effect according to the preferred frame correction as derived by Møller, assuming the existence of a preferred frame of reference moving with $v \approx 370 \mathrm{~km} / \mathrm{s}$ w.r.t. Earth, plotted as a function of the angle between $\vec{u}\left(\vec{v}=\vec{u} / u^{0}\right)$ and the parallel velocities of the emitter and absorber of radiation $V_{1} \approx 25 \mathrm{~m} / \mathrm{s}, V_{2} \approx 3 \mathrm{~m} / \mathrm{s}$ (Moller). A constant value (no directional dependence) predicted by STR / PF is plotted for comparison $(\mathbf{S T R}=\mathbf{P F})$

should be observed according to STR (two transformations combined in Eq. (24) cancel out when $\vec{u}=0$ and $\vec{V}_{1}= \pm \vec{V}_{2}$ ).

In a general case, as long as $\vec{u}$ is comparable to $\vec{V}$ the correction expected by Møller should give a significant contribution to the Doppler effect, depending on the direction of motion of the emitter (absorber). The above equations imply changes in observed frequency related to the change of the angle between $\vec{u}$ and $\vec{V}$. In particular, one would expect periodical changes caused by the orbital and rotational motion of Earth.

For a quantitative discussion we assume that the PF is associated with the cosmic background radiation frame; the speed of the solar system with respect to it is $v_{C M B}=(369.82 \pm 0.11) \mathrm{km} / \mathrm{s}$ [18]. Meanwhile, the Earth has an orbital speed of about $30 \mathrm{~km} / \mathrm{s}$, and a tangential speed related to rotation of less than $0.5 \mathrm{~km} / \mathrm{s}$. If the measurements are performed over a reasonably short period of time $\vec{u}$ can be considered constant to a good approximation. Meanwhile, the motion of the experimental setup constantly changes the direction of $\vec{V}$ and thus the angle between these two vectors. Mössbauer rotor experiments are sensitive only to the component of $\vec{u}$ in the plane of the rotating disk, thus the experiment has to be performed in several orientations of the setup in order to determine the complete $\vec{u}$ vector.

The Doppler shift calculated assuming $v_{C M B}$ and the conditions of the experiment by Turner and Hill [14] $\left(\vec{V}_{1} \approx 25 \mathrm{~m} / \mathrm{s}\right.$ parallel to $\left.\vec{V}_{2} \approx 3 \mathrm{~m} / \mathrm{s}\right)$ is shown in Fig. 2 as a function of the angle between $\vec{u}$ and $\vec{V}$. In this case the correction expected by Møller, of the order of $10^{-10}$, is significantly larger than the standard Doppler shift, of the order of $10^{-14}$. 


\section{Summary}

The interpretation and verification of the special theory of relativity received a lot of attention in the past. However, many of the questions which caused heated debates at the beginning of the last century, still remain valid. In particular, a very promising alternative theory has been proposed in the quite recent works of Rembieliński [10-13]. It not only provides explanation of commonly known relativistic phenomena equivalent to that of STR, but also allows to formulate Lorentz-covariant quantum theory [13], with covariant position and spin operators that do not exist in the relativistic quantum theory based on STR.

It has been shown that these two theories cannot be distinguished from each other in the majority of experiments aiming at the verification of STR. We have demonstrated that, despite the suggestions of some authors, also the Doppler effect for light does not provide a suitable experimental method for this purpose. We have shown that the two theories are by construction exactly equivalent in case of kinematic effects if all the transformations are correctly taken into account.

A few measurements of the directional anisotropy of the Doppler effect have been performed in the past, well before a proper relativistic theory preserving absolute simultaneity was formulated, and their results have been in agreement with the predictions of STR. It has to be noted that the strong upper limits imposed on the velocity of Earth with respect to a supposed preferred frame of reference, which seem to make the concept of PF strongly disfavored, are not justified. They have been derived from the experimental results using theoretical predictions, which do not present a viable alternative to STR, due to their inconsistence in introducing the anisotropy of the speed of light without taking into account the change of synchronization convention.

Further experimental searches in this field would be highly anticipated. In light of the indistinguishability of the two theories based on the kinematic effects there seem to be two ways of discriminating between them: (i) detection of tachyonic particles, which cannot be consistently described within STR, (ii) searches for the difference in relativistic quantum observables.

The first possibility, related to a possible tachyonic nature of a neutrino, is currently under investigation. Theoretical studies show that in such case the predicted beta decay spectrum differs from the predictions for a massive neutrino [19]. Furthermore, in the experiments performed until now an excess of counts near the end of the spectrum has been observed, which supports the hypothesis of a tachyonic neutrino. However, the precision of the measurement does not allow for a conclusive discrimination of this concept. This problem is one of the objectives of the ongoing KATRIN experiment [20], which should yield more precise results.

The relativistic quantum theory with absolute synchronization and the standard relativistic quantum mechanics give different predictions for some non-local phenomena, which opens a second possibility to discriminate between the two theories. In particular, investigating quantum spin observables for relativistic particles 
with mass seems promising due to a difference in the spin operators. An experiment investigating quantum spin correlations with a pair of massive particles has been proposed [21], however, up to our best knowledge there is no known way of preparing a pure singlet state at ultra-relativistic energies. A more practical approach is related to the study of quantum spin correlations in relativistic Møller scattering of polarized electrons [22], and an experiment of this kind is as well ongoing [23].

Acknowledgements We would like to thank Prof. J. Rembieliński and Prof. J. Ciborowski for inspiring discussions. This work was supported by a National Science Centre Grant No. 2017/25/N/ ST2/00619.

Open Access This article is licensed under a Creative Commons Attribution 4.0 International License, which permits use, sharing, adaptation, distribution and reproduction in any medium or format, as long as you give appropriate credit to the original author(s) and the source, provide a link to the Creative Commons licence, and indicate if changes were made. The images or other third party material in this article are included in the article's Creative Commons licence, unless indicated otherwise in a credit line to the material. If material is not included in the article's Creative Commons licence and your intended use is not permitted by statutory regulation or exceeds the permitted use, you will need to obtain permission directly from the copyright holder. To view a copy of this licence, visit http://creativecommons.org/licen ses/by/4.0/.

\section{References}

1. Michelson, A.A., Morley, E.W.: On the relative motion of the earth and the luminiferous ether. Am. J. Sci. 34, 333 (1887)

2. Liberati, S., Maccione, L.: Lorentz violation: motivation and new constraints. Ann. Rev. Nucl. Part. Sci. 59, 245 (2009)

3. Liberati, S.: Tests of Lorentz invariance: a 2013 update. Class. Quant. Grav. 30, 133001 (2013)

4. Reichenbach, H.: Axiomatization of the Theory of Relativity. University of California Press, Berkeley (1969)

5. Einstein, A., Podolsky, B., Rosen, N.: Can quantum-mechanical description of physical reality be considered complete? Phys. Rev. 47, 777 (1935)

6. Mansouri, R., Sexl, R.U.: A test theory of special relativity: I. Simultaneity and clock synchronization. Gen. Rel. Gravit. 8, 497 (1977)

7. Mansouri, R., Sexl, R.U.: A test theory of special relativity: II. First order tests. Gen. Rel. Gravit. 8, 515 (1977)

8. Mansouri, R., Sexl, R.U.: A test theory of special relativity: III. Second-order tests. Gen. Rel. Gravit. 8, 809 (1977)

9. Reinhardt, S., et al.: Test of relativistic time dilation with fast optical atomic clocks at different velocities. Nat. Phys. 3, 861 (2007)

10. Rembieliński, J.: The relativistic ether hypothesis. Phys. Lett. A78, 33 (1980)

11. Rembieliński, J., Włodarczyk, M.: "Meta" relativity: against special relativity? arXiv:1206.0841 (2012)

12. Rembieliński, J.: Tachyons and preferred frames. Int. J. Mod. Phys. A 12, 1677 (1997)

13. Caban, P., Rembieliński, J.: Lorentz-covariant quantum mechanics and preferred frame. Phys. Rev. A 59, 4187 (1999)

14. Turner, K.C., Hill, H.A.: New experimental limit on velocity-dependent interactions of clocks and distant matter. Phys. Rev. 134, 252 (1964)

15. Champeney, D.C., Moon, P.B.: Absence of Doppler shift for gamma ray source and detector on same circular orbit. Proc. Phys. Soc. 77, 350 (1961) 
16. Champeney, D.C., Isaak, G.R., Khan, A.M.: An 'aether drift' experiment based on the Mössbauer effect. Phys. Lett. 7, 241 (1963)

17. Møller, C.: New experimental tests of the special principle of relativity. Proc. R. Soc. Lond. A 270, 306 (1962)

18. Akrami, Y., et al. (Planck Collaboration): Planck 2018 results. I. Overview and the cosmological legacy of Planck. arXiv:1807.06205 (2018)

19. Ciborowski, J., Rembieliński, J.: Tritium decay and the hypothesis of tachyonic neutrinos. Eur. Phys. J. C 8, 157 (1999)

20. Osipowicz, A., et al. (KATRIN collaboration): KATRIN: A next generation tritium beta decay experiment with sub-eV sensitivity for the electron neutrino mass. arXiv:hep-ex/0109033 (2001)

21. Rembieliński, J., Smoliński, K.A.: Quantum preferred frame: does it really exist? EPL 88, 10005 (2009)

22. Caban, P., Rembieliński, J., Włodarczyk, M.: Møller scattering and Einstein-Podolsky-Rosen spin correlations. Phys. Rev. A 88, 032116 (2013)

23. Ciborowski, J., et al.: A project to measure quantum spin correlations of relativistic electron pairs in Møller scattering. EPJ Web Conf. 164, 01004 (2017)

Publisher's Note Springer Nature remains neutral with regard to jurisdictional claims in published maps and institutional affiliations. 